\title{
Caries and collaborations in context
}

\author{
Nigel B. Pitts ${ }^{1}$
}

\section{Key points}

Despite a lack of incentives, individuals and organisations are often motivated to collaborate towards 'making the world a better place'. To be successful, such collaborations typically need to be long-term.
In the caries world, the Global Collaboratory for Caries Management, run from King's College London, has been designed as collaborative and structure-based on best evidence and consensus.
The Global Collaboratory for Caries Management also brings together two charities: the International Caries Detection and Assessment System Foundation, developing resources to aid dental practitioners, educators and teams; and the Alliance for a Cavity-Free Future, operating multiple international advocacy and policy initiatives.

\begin{abstract}
Dental caries is the most prevalent non-communicable disease globally and affects all age groups across the life course. Caries (and the dental cavities it produces) create very significant personal, societal and economic burdens across the world, despite dental cavities being largely preventable. This is why so many individuals and groups are collaborating to do something to improve the world landscape of avoidable cavities. Over the past 20 years, a series of international partnerships have been developed in caries research, education and management, which have paved the way for a shift towards collaborative, inter-disciplinary and multi-stakeholder approaches to caries. This paper: 1) discusses the motivation for individuals and organisations to collaborate towards 'service to society'/'making a difference'/'making the world a better place'; 2) explains that collaboration to make an impact is undeniably complicated, but highly desirable; 3 ) suggests that successful collaborations typically need to be sustainable and longterm; 4) provides some examples from the caries world of the range and depth of collaborations making a difference; and 5) suggests that we now seem to be at multiple 'tipping points' to accelerate the progress of integration, intersectoral and interprofessional collaboration and of aligning caries control with the broader context of oral health and general health globally.
\end{abstract}

\section{Introduction}

Dental caries is the most prevalent noncommunicable disease (NCD) globally and affects all age groups across the life course. ${ }^{1}$ Caries (and the dental cavities it produces) create very significant personal, societal and economic burdens across the world. Some 2.3 billion people have untreated cavities in permanent teeth and over 530 million children suffer from untreated cavities of the primary teeth. Despite dental cavities being largely preventable, untreated cavities in permanent teeth are ranked as number one for prevalence

'Faculty of Dentistry, Oral and Craniofacial Sciences, King's College London, Tower Wing, Guy's Hospital, London, SE1 9RT, UK.

Correspondence to: Nigel B. Pitts

Email address: nigel.pitts@kcl.ac.uk

Refereed Paper.

Accepted 20 October 2021

https://doi.org/10.1038/s41415-021-3731-3 in the entire 'Global Burden of Disease Study' across all 291 diseases and injuries considered. ${ }^{1}$ This is why so many individuals and groups are doing something to improve the world landscape of avoidable cavities. The good news is that they are increasingly collaborating to make an impact together.

King's College London's 'Strategic Vision $2029^{22}$ lays out a map towards linking appropriate research and the identification of best evidence, synthesised with international partners, to share in educational and policy developments to see improvements to health and wellbeing, both locally and globally; simply put, outlining the need to work together to 'make the world a better place. Caries can provide one exemplar of this approach. Over the past 20 years, a series of international collaborations have been developed in caries research, education and management, which have paved the way for a shift towards interdisciplinary and multi-stakeholder approaches to caries prevention and control.

\section{Motivation to collaborate for 'service to society'/'making the world a better place'}

The motivations around what leads organisations, teams and individuals to collaborate around these common purposes are increasingly being explored as the phenomenon becomes more common, across, for example, universities, companies, health services, academics and health professionals. Groups often collaborate because they are united by a desire to make a difference and improve a diverse range of challenges in order to contribute to making elements of the world they value a better place. A recent report exploring academic incentives and research impact examined reward and recognition systems for staff. The report unfortunately found that 'for the most part, measures of research impact on societal problems are missing in action from performance evaluation 
criteria within academic disciplines.' It revealed a series of unintended consequences around current academic incentives on research. It is to be hoped that these issues can be rectified. Many academics and health professionals (and the organisations they work in) elect to collaborate to achieve societal impact because it fits with their ethos and professional values. Similarly, organisations are becoming more clearly value-driven and perceive reputational and market advantage in such corporate and social responsibility activities.

\section{Collaboration to make an impact is undeniably complicated but highly desirable}

The central values and impact objectives of an organisation can be made clear at a high level (that is, in general terms), but there is a real challenge in being specific, as the detail of specific beneficial changes (impact) and how to make a difference 'at the front lines' is different across disciplines, countries and cultures. Intimate working knowledge of the specific area under consideration is needed to plan and achieve beneficial societal or economic impact; therefore, the collaborations and actions must be tailored 'glocally' (using global values and evidence but adapted for local use). The understanding of the word 'impact' is also a complication, as there is widespread confusion between research impact and service and education, as well as local versus global impact. The boundaries are not clear-cut, as there is often vast overlap between these areas with multifaceted impacts.

The Global Collaboratory for Caries Management (GCCM) at King's College London (Fig. 1) was established in 2013 and is an example of pursuing a collaborative approach against caries, drawing together multiple projects, charities and groups and working with local and international partners to see improvements in oral and wider health and wellbeing, both at a practice and policy level. GCCM initiatives span multiple countries and domains to collaboratively push for beneficial changes around a common purpose; to achieve improvements in society, health and care, without reward.

Although the three core elements of the GCCM are complementary, the type of collaborations needed for the International Caries Classification and Management System (ICCMS) (https://www.iccms-web.

An 'umbrella group' facilitating the delivery of Preventive Dental Medicine for caries

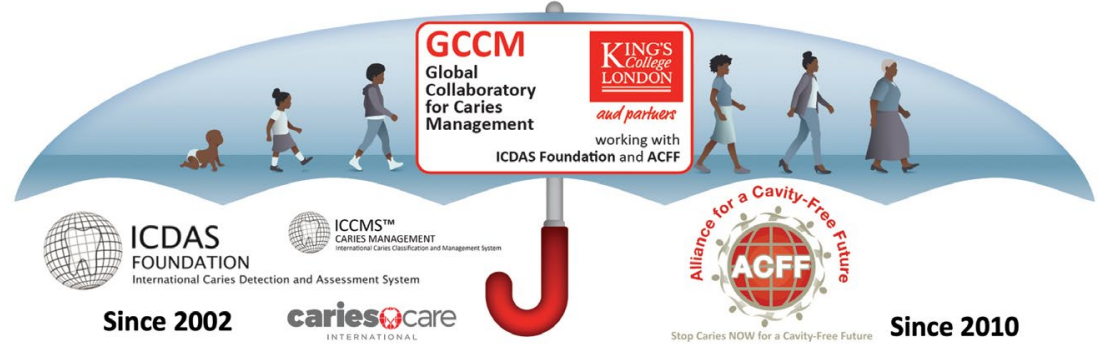

Fig. 1 The Global Collaboratory for Caries Management. Logo and illustrations reproduced with permission from ACFF; logos reproduced with permission from ICDAS Foundation, CariesCare International and King's College London

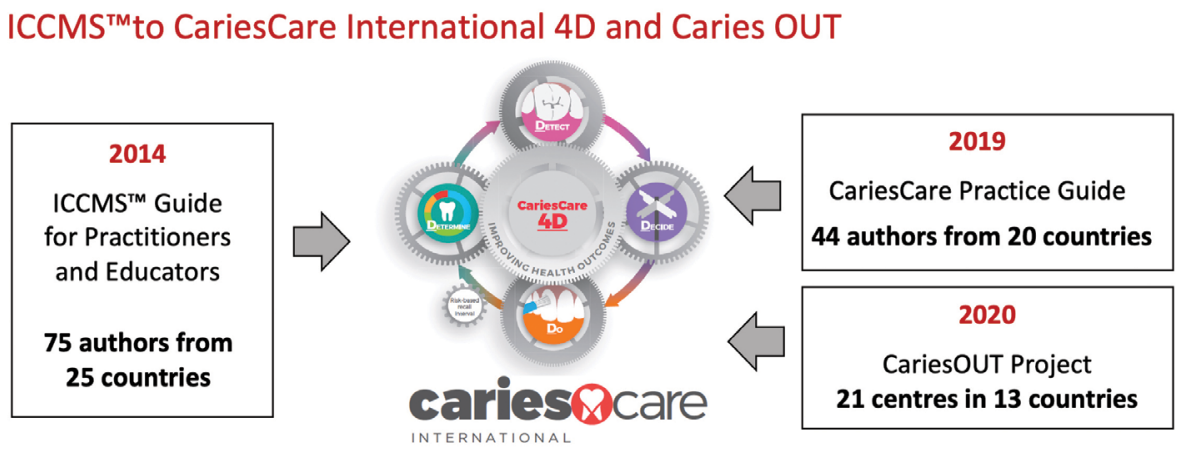

Fig. 2 Collaborations since 2014. Logo reproduced with permission from CariesCare International

com/), CariesCare International (https:// cariescareinternational.com/) and the Alliance for a Cavity-Free Future (https://www. acffglobal.org/) are significantly different.

\section{Successful collaborations typically need to be sustainable and long- term}

Some of the key requirements for managing a successful collaboration are:

- A trusted culture and a 'safe space' in which to collaborate

- Running collaborations as a team sport but recognising that some key individuals and their networks are also valuable

- Taking a long-term view, as opposed to endlessly 'reinventing the wheel'

- Appreciating that a long-term history of a collaboration may be invisible to, or not understood by, other stakeholders

- Resilience to be able to survive changes in key individuals

- Awareness of the challenges associated with individual or corporate egos and a default position of many in protecting their own interests
- Competitiveness of organisations and individuals linked to short-termism.

A volunteer core group of 20 people from many countries developed the initial full International Caries Detection and Assessment System (ICDAS) criteria up to 2007. Figure 2 illustrates the different collaborations that have been developed by the ICDAS Foundation since 2013. A wider group of collaborators (with 75 authors from 25 countries) was needed to produce the ICCMS' 'Guide for Practitioners and Educators' published in 2014. ${ }^{4}$ When, in 2018, a practice-friendly version of the guidance was needed, a new volunteer group of external collaborators was included so that the 'CariesCare International Practice Guide' and illustrated case study ${ }^{6}$ were developed (by 44 authors from 20 countries). With the arrival of the COVID-19 pandemic, a new format of the guidance was required for treating children without aerosol generating procedures. This is being developed by another group as the 'Caries OUT' study, with a range of different collaborators from 21 dental centres in 13 countries. All of these individuals have contributed to the development and implementation of the current CariesCare International 4D system. 


\section{Exemplars from the caries world of the range and depth of collaborations making a difference}

The Alliance for a Cavity-Free Future (ACFF) is a globally reaching, non-profit organisation, which has been working since 2010 to localise initiatives for change through a collaborative network of dental and public health professionals, educators, organisations and industry partners, as well as other related and interested parties. The ACFF is guided by an international expert panel, with current members representing multiple dental schools and organisations across 11 different countries. ACFF operates initiatives both at a grass roots level and a policy level and there are currently 29 ACFF Chapters working to target locally appropriate interventions and initiatives across over 40 countries, with a UK Chapter the latest to launch in 2021. A 2019 survey of Chapter activities showed that across dentists, other health professionals and the public, over 700,000 people were reached through an annual caries awareness day alone, with over 8.6 million people reached in 2019 overall.

The ACFF Caries Puzzle (Fig. 3) demonstrates the need to join up stakeholders to create collaborative action towards a cavityfree future, as caries remains a multifactorial disease. The 'Caries Puzzle' concept was developed in 2016 when the ACFF held a 'World Caries Summit' in Dubai, where an international panel of experts reviewed scientific evidence in their own areas of study and laid out the need to collaborate and work across what are frequently seen as silos.

ACFF also has many examples of effective international collaborations across: education ${ }^{8,9}$ (also using the Caries Puzzle); against early childhood caries - with the International Association for Paediatric Dentistry producing and disseminating the 'Bangkok Declaration'; ${ }^{10,11}$ creating consensus on a regional basis - facilitating a comprehensive consensus on all aspects of caries for Latin American and Caribbean countries ${ }^{12}$ and; driving policy development - with the 'Dental Policy Lab' initiative launched in 2017, drawing together a multi-disciplinary, international, collaborative group including practitioners, academics, public health professionals, governmental representatives and Chief Dental Officers, industry representatives and health economists, to tackle some of the key issues faced when pushing for a policy shift towards preventive dental systems. ${ }^{13,14,15}$

\section{We now seem to be at multiple 'tipping points'}

Progress with improving caries prevention and management at both the individual and public health levels has been frustratingly slow over the last decades. However, it seems that we might be approaching an acceleration of progress as there appear to be a series of multiple tipping points. Perhaps the most obvious is the COVID-19 pandemic, which has accelerated and encouraged change and how it can be contemplated. At the time of writing, there now seems to be an increasing appetite for using evidence and for all forms of prevention, as well as an increasing awareness of health inequality with a desire to do something about it. There is also an increased interest in environmental and green issues around the climate and sustainability (linking to avoiding amalgam or plastic restorations) and an increased awareness of global aspects of health and global thinking. These shifts in public and political views can make long-needed changes now possible, having demonstrated that unusual alliances can be effective and welcome and that government/ private partnerships can be effective if properly managed and have given increased prominence for the World Health Organisation (WHO).

So, it seems that the pieces of the caries puzzle are at last aligning ${ }^{16}$ and that it is time to change the narrative of the caries and oral health story ${ }^{17}$ to one of joining hands, of integration and of intersectoral and interprofessional collaboration.

Figure 4 shows the recent report of the ACFF Making Cavities History Taskforce ${ }^{1}$ which

\section{Joining up stakeholders to create collaborative action towards a cavity-free future}

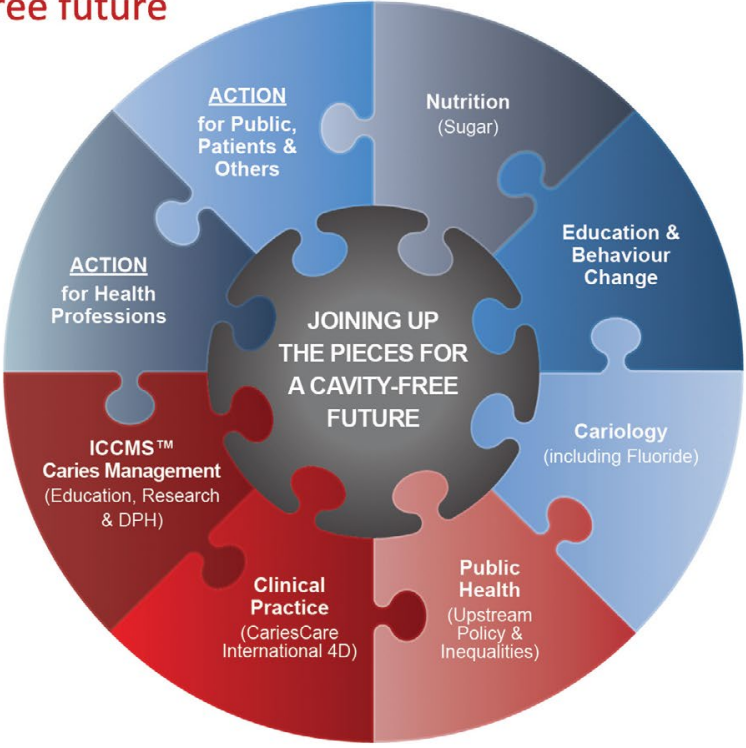

Fig. 3 The ACFF Caries Puzzle. Illustration reproduced with permission from ACFF

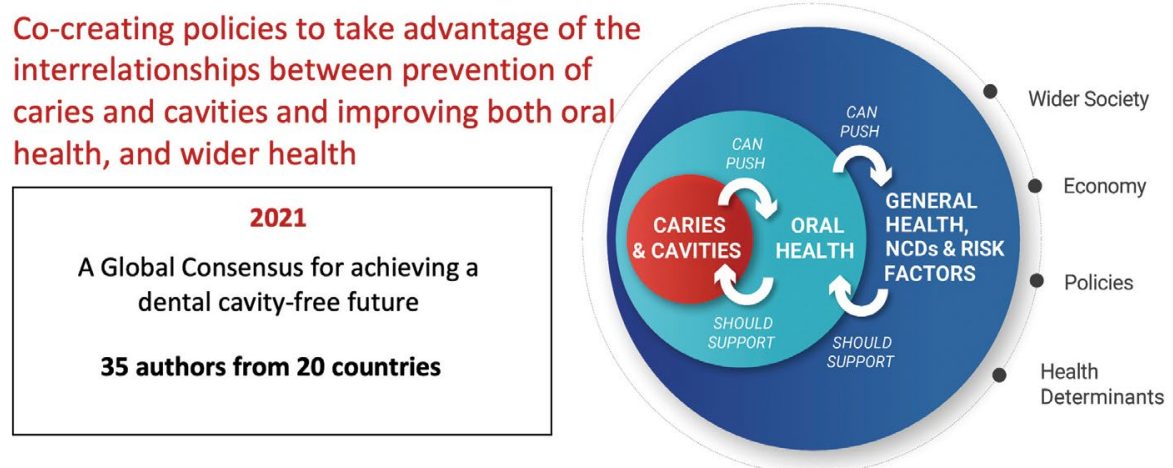

Fig. 4 The ACFF Making Cavities History Taskforce. Illustration reproduced with permission from ACFF 
brought together 35 participants from 20 countries to co-create a consensus on policies for caries prevention, taking advantage of the interrelationships between prevention of caries and cavities and improvements in both oral and wider health. By including caries and oral health in the wider $\mathrm{NCD}^{18}$ discussion, we now have policies ${ }^{19}$ to go further towards reducing the unacceptable burden of cavities and to help improve general health through wider collaborations in prevention and care across public health and the health professions.

The FDI World Dental Federation is now predicting that 'by 2030 , oral health professionals will collaborate with a wide range of health workers to deliver sustainable, healthneeds-based and people-centred healthcare. ${ }^{20}$ The chances of this happening have been dramatically increased by the first WHO 'Resolution on oral health' for 14 years, which was approved by the World Health Assembly in May 2021. ${ }^{21}$

\section{Conclusion}

Dental caries is an important burden but should no longer be seen as a standalone issue, but one that shares common risk factors with many other NCDs. As such, it should be addressed in conjunction with wider oral and general health policy and care delivery to ensure maximum health benefit is achieved across the board, ${ }^{22}$ a view that has been expressed by the late Aubrey Sheiham for decades. The collaborations explored over the last two decades of progress towards creating a future free from the burden of dental cavities have been extensive, multifaceted and beneficial. Individuals and organisations have generously collaborated over extended periods in the 'service of society'. If we are to continue to make progress towards 'making cavities history', we must use the opportunities presented by the current multiple 'tipping points' to accelerate the progress and continue the push of developing a narrative of joining hands, of integration, of intersectoral and interprofessional collaboration and of aligning caries control with the broader context of oral health and general health globally.
Ethics declaration

Nigel B. Pitts is founder of the Global Collaboratory for Caries Management at King's College London, Faculty of Dentistry, Oral and Craniofacial Sciences (FoDOCS). He a is founding chair of the ICDAS Foundation charity and a member of the CariesCare International Executive. He is also co-founder and global chair of the Alliance for a Cavity-Free Future charity. With exception of the above, Nigel B. Pitts declares no potential conflicts of interest with respect to the research, authorship and/or publication of this article.

Author contributions

Nigel B. Pitts conceived and drafted the manuscript.

\section{Acknowledgements}

The author acknowledges the support of an extensive collaborative network in the long-term development of this work. These include, but are not limited to: colleagues in research, education and service at King's College London as well as King's College London's Partners in the Global Collaboratory for Caries Management; the ICDAS Foundation Board and Coordinating Committee; the ICCMS Guidance authors; the CariesCare International Guide authors; the Alliance for a Cavity-Free Future, its chapter teams, global expert panel, Making Cavities History Taskforce and supporters; and all other supporters - individuals, universities, organisations, sponsors and collaborators. The author also wishes to thank Catherine Mayne at the Alliance for a Cavity-Free Future for input and practical support with the preparation of the manuscript and figures.

\section{References}

1. Pitts N, Mayne C. A Global Consensus for achieving a dental cavity-free future. 2021. Available at https:// $\mathrm{kclpure.kcl.ac.uk/portal/en/publications/a-global-}$ consensus-for-achieving-a-dental-cavityfreefuture(c3b4777e-f615-4a5e-934d-e47a11843183).html (accessed November 2021).

2. King's College London. King's Strategic Vision 2029 2017. Available at https://www.kcl.ac.uk/about/ strategy (accessed November 2021).

3. Academy Health Paradigm Project. Academic Incentives and Research Impact: Developing Reward and Recognition Systems to Better People's Lives, Horizon Scan. 2021. Available at https://academyhealth.org/sites/default/files/ publication/\%5Bfield_date\%3Acustom\%3AY\%5D$\% 5$ Bfield_date $\% 3$ Acustom $\% 3$ Am $\% 5$ D/ academicincentivesresearchimpact_feb2021.pdf (accessed November 2021).

4. Pitts N B, Ismail A I, Martignon S et al. ICCMS ${ }^{\mathrm{TM}}$ Guide for Practitioners and Educators. 2014. Available at https://iccms-web.com/uploads/ asset/59284654c0a6f822230100.pdf (accessed June 2021).
5. Martignon S, Pitts N B, Goffin G et al. CariesCare practice guide: consensus on evidence into practice. BrDent J 2019; 227: 353-362.

6. Beltran E O, Guiu L, Zarta O L, Pitts N B, Martignon S. Caries classification and management in the context of the CariesCare International (CCI) consensus: a clinical case study. Br Dent J 2019; 227: 363-366.

7. Martignon S, Cortes A, Douglas G V A et al. CariesCare International adapted for the pandemic in children: Caries OUT multicentre single-group interventional study protocol. BMC Oral Health 2021; 21: 329.

8. Pitts N B. The shape of the future of dental education for dental cariesand how we get there: Introduction to the ACFF Caries Workshop. Eur J Dent Educ 2018; DOI: 10.1111/eje.12344

9. Pitts N B, Mazevet M E, Mayne C, Shaping the Future of Dental Education Cariology Group. Shaping the future of dental education: Caries as a case-study. Eur J Dent Educ 2018; DOI: 10.1111/eje.12345.

10. Tinanoff N, Baez R J, Diaz Guillory C et al. Early childhood caries epidemiology, aetiology, risk assessment, societal burden, management, education, and policy: Global perspective. Int J Paediatr Dent 2019; 29: 238-248.

11. International Journal of Paediatric Dentistry. Early Childhood Caries: IAPD Bangkok Declaration. Int I Paediatr Dent 2019; 29: 384-386

12. Sampaio F C, Bönecker M, Paiva S M et al. Dental caries prevalence, prospects, and challenges for Latin America and Caribbean countries: a summary and final recommendations from a Regional Consensus. Braz Oral Res 2021; DOI: 10.1590/1807-3107bor-2021.vol35.0056.

13. Pitts N, Mazevet M, Mayne C et al. Towards a Cavity Free Future: How do we accelerate a policy shift towards increased resource allocation for caries prevention and control? 2017. Available at https://www.kcl.ac.uk/ policy-institute/assets/towards-a-cavity-free-future. pdf (accessed November 2021).

14. Pitts N, Mazevet M, Mayne C, Boulding H, Pow R. Towards paying for health in dentistry: How can we create and implement acceptable prevention-based dental payment systems to achieve and maintain health outcomes? 2019. Available at https://kclpure.kcl.ac.uk/ portal/en/publications/towards-paying-for-health-indentistry(01711f4e-5a70-4762-b72e-2c2af41ce589). html (accessed November 2021).

15. Pitts N, Pow R. Towards Oral and Dental Health through Partnership: How can the oral health and dental industries benefit from enabling positive behaviour in caries prevention and control among patients and the public? 2020. Available at https://kclpure.kcl.ac.uk/ portal/en/publications/towards-oral-and-dentalhealth-through-partnership(ab92fcac-8cdb-4f06-989573bbe0e3972e).html (accessed November 2021).

16. Pitts N. The pieces of the caries puzzle align. Br Dent J 2021; 230: 493.

17. Benzian H. Time to change the narrative. Br Dent J 2021; 230: 623.

18. Pitts N, Twetman S, Fisher J, Marsh P. Understanding caries as a non-communicable disease. Br Dent J 2021; 749-753.

19. 19. Pitts N B, Mayne C. Making Cavities History: A Global Policy Consensus for Achieving a Dental CavityFree Future. JDR Clin Trans Res 2021; 6: 264-267.

20. Glick M, Williams D M, Ben Yahya I et al. Vision 2030: Delivering Optimal Oral Health for All. 2021. Available at https://www.fdiworlddental.org/vision2030 (accessed November 2021).

21. World Health Assembly. Oral Health. 2021.Available at https://apps.who.int/gb/ebwha/pdf_files/WHA74/ A74_R5-en.pdf (accessed June 2021).

22. Sheiham A, Watt $R G$. The common risk factor approach a rational basis for promoting oral health. Community Dent Oral Epidemiol 2000; 28: 399-406. 